\title{
Una aproximación a la gestión de la diversidad en el Perú
}

\author{
Rosa María Fuchs Ángeles \\ International M.B.A. por el Instituto de Empresa Business School, Madrid-España. \\ Profesora e investigadora del Departamento Académico de Administración de la Universidad del Pacífico, \\ Lima, Perú.
}

\section{Resumen}

La gestión de la diversidad ha cobrado importancia en las empresas del primer mundo, pues un grupo humano diverso puede aportar ventajas a las organizaciones. Sin embargo, aún encontramos en nuestra realidad cercana empresas en las que las mujeres son consideradas como ciudadanos de segunda categoría, y en las que ser una persona mayor de 50 años puede significar perder el empleo por los estereotipos asociados a la edad. En este artículo se presenta la gestión de la diversidad como imperativa para las empresas del siglo XXI, aunque se reconocen también sus riesgos asociados. En el marco teórico se muestran los ámbitos de la diversidad y las principales prácticas que se desarrollan para gestionarla de manera adecuada. Luego, se presentan los resultados obtenidos de las entrevistas con cuatro empresas que operan en el Perú y que gestionan la diversidad. Finalmente, se presentan las conclusiones del estudio.

Palabras clave: Gestión de la diversidad, diversidad cultural, diversidad de género, diversidad de edad.

\section{Introducción}

Los líderes del siglo XXI enfrentan un entorno competitivo en el que la gestión de las empresas no es sencilla. Uno de los retos que afrontan es la diversidad de la fuerza laboral y las presiones de los diferentes stakeholders para incluir a los grupos minoritarios o tradicionalmente excluidos. En los países desarrollados, gestionar la diversidad forma parte de la cultura de las organizaciones y se ha tomado conciencia de que genera una ventaja competitiva. En nuestro país, sin embargo, la gestión de la diversidad aún se encuentra en una etapa inicial. La principal preocupación respecto del tema parece estar enfocada en la diversidad de género, lo que en nuestra realidad significa permitir que las mujeres tengan las mismas oportunidades que los hombres. En el presente artículo se presentan las venta- jas y desventajas de la diversidad, algunas recomendaciones para gestionarla de manera adecuada y la perspectiva de cuatro empresas que operan en el Perú y que han interiorizado este tema.

\section{Objetivos del estudio}

- Reconocer los ámbitos de la diversidad y entender qué significa gestionarla.

- Identificar el grado de importancia que cuatro empresas que operan en el Perú, y son reconocidas por su gestión de personas, le otorgan a la gestión de la diversidad

\section{Marco conceptual}

La diversidad se puede definir como cualquier diferencia percibida entre las personas en lo que 
se refiere a edad, género, profesión, orientación sexual, origen geográfico, estilo de vida, entre otras. La gestión o administración de la diversidad consiste en asegurar que existen factores que promueven una fuerza laboral diversa para lograr la máxima productividad (Mondy y Noe 2005).

Los gerentes del siglo XXI enfrentan el reto de coordinar esfuerzos con personal heterogéneo en términos de sexo, raza, edad, entre otros factores. A diferencia de décadas anteriores, en que las minorías representaban una proporción pequeña del personal y se suponía que querían adaptarse a las mayorías, hoy se acepta la idea que los trabajadores no abandonan sus valores culturales ni sus preferencias de estilo de vida en el centro de trabajo (Robbins y Coulter 2005). Por ello, la gestión de la diversidad se ha convertido en una prioridad.

La diversidad presenta beneficios y riesgos asociados (Mayo 2002). La variedad de habilidades, conocimientos e información de los miembros de la organización puede beneficiar la búsqueda de innovación, resolución de problemas y toma de decisiones. Otro factor positivo es el tipo de red de contactos que se puede asociar a un grupo diverso. Por otro lado, las diferencias pueden generar conflictos que implicarían que las personas abandonen las organizaciones y, por tanto, se incremente el nivel de rotación en estas últimas. Además, según Mayo (2002), los procesos psicosociales subyacentes de la diversidad pueden fomentar favoritismos hacia un grupo y originar su desunión.

La Dirección General de Empleo de la Comisión Europea publicó, a finales de 2003, un informe en el que se indica que las compañías que fomentan la diversidad tienen mejores resultados Mantener la palabra resultados. El artículo lo menciona sí, se refiere por ejemplo a clima organizacional) pues refuerzan la competitividad a largo plazo y los resultados a mediano y corto plazo (Morales 2006). La gestión de la diversidad puede significar una ventaja competitiva en, al menos, seis campos (Cox y Blake 1991): costos, adquisición de recursos, marketing, creativi- dad, solución de problemas y flexibilidad organizacional. Chemers et al. (1995) coinciden en que la diversidad fomenta la innovación; sin embargo, también muestran que podría generar problemas cuando existen trabajadores muy tradicionales que no muestran apertura hacia la diversidad. De Anca (2008) añade como ventaja la retención del talento, debido al otorgamiento de modalidades de trabajo flexibles y la generación de lealtad y motivación en los trabajadores.

\section{Ámbitos de la diversidad}

La diversidad se puede clasificar en diversidad cultural, de género y de edad, entre otros.

\section{Diversidad cultural}

La diversidad cultural se refiere a la relación de diferentes culturas existentes. En una organización, se ve reflejada en las creencias, los valores y las costumbres de los trabajadores, debido a que estos últimos son oriundos de diferentes ciudades o países. Esta diversidad tiene mayormente efectos positivos, tales como el fomento de la creatividad y el conocimiento de costumbres distintas. A manera de ejemplo, aunque la mayoría de trabajadores profese la religión católica, la empresa deberá considerar las festividades que otras religiones, como la judía, practican. Esto implica que se debe entender y permitir que un grupo de ellos pueda tener un día libre en un momento diferente al de la mayoría de personas. Una organización multicultural trata de estar libre de prejuicios porque estos generan discriminación (Ovalle y Marchant 2005). Asimismo, una organización multicultural se diferencia de una organización denominada "plural» cuando tiene un compromiso más profundo con la diversidad. Algunos factores para lograr el éxito en la gestión de la diversidad cultural son: el compromiso de las altas autoridades, el conjunto de programas de las áreas de Recursos Humanos alineados con la estrategia de diversidad corporativa y el verdadero convencimiento de que, gracias a la diversidad cultural, se generará valor agregado. 
Diversidad de género

La diversidad de género es una de las más populares en América Latina. El que la mujer se haya desempeñado como ama de casa de manera predominante ha originado que su incursión, cada vez mayor, en el mercado laboral se encuentre en estudio permanente. Incluso, se ha llegado a acuñar el término «techo de cristal» para referirse a las barreras invisibles que impiden a las mujeres bien preparadas acceder a niveles de dirección elevados (Ramos 2005). A lo largo de la historia, la mujer ha desempeñado trabajos productivos no remunerados o de baja calidad (Alles 2000); sin embargo, esta situación ha empezado a cambiar. Aun más, según Halladay y Thomas (2002), el hecho de que las mujeres hayan estado dedicadas a la crianza de los hijos y a la administración de recursos, las ha capacitado para ser innovadoras y emprendedoras. Por lo tanto, ha generado una oportunidad para su surgimiento como empresarias.

Pese al cambio que se está viviendo en cuanto a la participación de la mujer en el mercado laboral, las funciones tradicionales de género siguen enraizadas en América Latina, lo que restringe su movilidad en la empresa (Piras 2006). Según Maxfield (2007), las mujeres no están bien representadas en los altos niveles jerárquicos. De acuerdo con un estudio realizado en 2004 , en 75 compañías de América Latina (Maxfield 2007), solo el $10 \%$ de los cargos de presidentes o vicepresidentes eran ocupados por mujeres. En opinión de Barbera et al. (2002), las compañías deberían saber aprovechar el potencial de las mujeres en habilidades de comunicación, relaciones interpersonales, capacidad para manejar el estrés e innovación.

Cuando se menciona la diversidad de género, se plantea la idea de crear espacios donde hombres y mujeres puedan brindar aportes en función de sus cualidades y, a la vez, aprovechar las oportunidades en equidad.
Diversidad de edad

La percepción que se tiene en el ámbito laboral sobre las personas mayores es que son menos responsables, menos flexibles a los cambios organizacionales, difíciles de entrenar y costosas. Sin embargo, las personas de mayor edad son las que poseen la menor tasa de ausentismo (Roscigno et al. 2007). Las buenas prácticas en materia de diversidad de edad se refieren a que cualquier individuo reciba el apoyo para alcanzar su potencial, sin que la edad represente una desventaja (Walker 1999).

Se deben reconocer las características de los individuos según la etapa de la vida en la que se encuentran, para así poder identificar los aportes que están en condiciones de brindar a las organizaciones. Los empleados menores de 35 años están deseosos de ocupar cargos de responsabilidad y, al mismo tiempo, son menos leales a las instituciones. Los que se encuentran entre los 35 y 54 años son más autoritarios e idealistas. Si el trabajo les resulta satisfactorio moverán montañas; de lo contrario, serán improductivos. Los empleados mayores de 55 años confían en la autoridad, respetan las reglas y son leales a las instituciones. Asimismo, socializan más que los jóvenes, por lo que posiciones como las de call centers son buenas para ellos (Manaut 2006).

\section{Iniciativas para gestionar la diversidad}

Algunas de las iniciativas que se destacan en la gestión de la diversidad son:

- La flexibilidad. Existen compañías que otorgan las facilidades necesarias para que su personal pueda trabajar a distancia o en jornadas reducidas. Mediante esta modalidad las madres trabajadoras pueden combinar sus responsabilidades de madres y profesionales, de manera que las mujeres no abandonen sus puestos de trabajo por su condición de madres. 
- La información. Las empresas que aseguran que la diversidad es una ventaja competitiva brindan información, de manera permanente, sobre lo que realmente significa la diversidad y sus beneficios. Asimismo, ilustran los comportamientos discriminatorios con el fin que estos se eviten. Es usual que estos programas reciban el apoyo directo de la alta dirección.

- Prácticas de reclutamiento de personal. La búsqueda de los futuros trabajadores de la empresa se realiza en diferentes ciudades. A su vez, los diversos profesionales provendrán de universidades diferentes. Esto va de la mano con programas de adaptación cultural para los miembros de la empresa.

- Políticas establecidas. Algunas empresas implementan políticas y códigos que contemplan medidas de no discriminación a grupos minoritarios; incluso, especifican cómo se procederá de detectarse problemas de este tipo.

- El área responsable. Si bien la gestión de la diversidad se inicia en el compromiso de la alta dirección, es necesario que haya un área responsable de mantener los diferentes programas. Por lo general, es el área de Recursos Humanos.

- Planes de carrera. Desarrollar planes de carrera con los trabajadores, tanto con los jóvenes como con los de edad avanzada, contribuirá a la satisfacción del empleado y a la identificación de las mejores áreas. De esa manera, los trabajadores podrán ser más productivos.

- Grupos de trabajo mixtos. Los grupos de trabajo conformados por hombres, mujeres y personas de diferentes edades contribuyen a que los miembros de la empresa valoren más la diversidad; así como, a la transferencia de conocimiento y experiencia.

Fórmulas como la flexibilidad horaria, el teletrabajo, brindar facilidades de guardería, son algunos de los instrumentos que las grandes empresas utilizan para gestionar la diversidad (De Anca 2008).

Existen condiciones que permiten que las diferencias de los grupos estén al servicio del aprendizaje, el crecimiento y la renovación de la empresa (Thomas y Ely 1999):
- La alta dirección debe comprender que una fuerza de trabajo diversificada abarca enfoques y perspectivas sobre el trabajo muy diferentes, razón por la cual debe valorar realmente la variedad de ideas y opiniones.

- La alta dirección debe reconocer tanto las oportunidades de aprendizaje, como los retos que proporciona la expresión de las distintas perspectivas existentes para la empresa.

- La cultura de la empresa debe crear una expectativa de altos niveles de rendimiento para todos.

- La cultura de la empresa debe estimular el desarrollo personal.

- La cultura de la empresa debe promover la franqueza.

- La cultura debe lograr que los empleados se sientan valorados.

\section{Metodología}

Durante el primer semestre de 2009, la Facultad de Administración y Contabilidad de la Universidad del Pacífico ofreció el Taller de Investigación denominado «Diversidad como estrategia de gestión». En él, los alumnos de pregrado levantaron información de fuentes secundarias sobre la gestión de la diversidad en la empresa global y tres ámbitos de la diversidad: cultural, de género y de edad. Luego de realizar un análisis de la información encontrada, se elaboró un cuestionario que se aplicaría a empresas peruanas que estuvieran trabajando el tema de diversidad. El cuestionario tuvo como finalidad conocer el grado de importancia que las organizaciones le otorgan a la gestión de la diversidad e identificar sus principales prácticas. Se aplicaron cinco encuestas a los encargados de Recursos Humanos de las empresas. Sin embargo, uno se descartó por no cumplir con los estándares correspondientes durante su aplicación.

\section{La diversidad en cuatro empresas peruanas}

Los cuestionarios se aplicaron a cuatro empresas que operan en el Perú. Dos de ellas pertenecen al rubro de la consultoría; una, al de tecnología y la última, al de consumo masivo. La sede de la 
casa matriz de dichas empresas se encuentra en el hemisferio norte.

A continuación se presentan los principales resultados. Las respuestas se encuentran clasificadas según el sector económico al que pertenece la empresa.

\section{Concepto de diversidad}

Las empresas encuestadas reconocen que la diversidad involucra distintos ámbitos y solo una de ellas la menciona como una fuente de ventaja competitiva (véase el cuadro 1). Se puede observar que el respeto a las diferencias es el denominador común.

\section{Origen de la filosofía de diversidad}

En tres de las cuatro empresas encuestadas, la filosofía de gestionar la diversidad no surgió en el Perú sino que proviene de la casa matriz (véase el cuadro 2). Este resultado nos hace reflexionar sobre la etapa inicial en la que se encuentra la gestión de la diversidad en el Perú.

\section{Cuadro 1}

\section{Concepto de diversidad}

\begin{tabular}{|l|l|}
\hline \multicolumn{1}{|c|}{ Empresas por sector } & \multicolumn{1}{c|}{ Definición de diversidad } \\
\hline Consultoras en auditoría (2) & $\begin{array}{c}\text { - La diversidad es la mezcla variada de nuestros recursos humanos, } \\
\text { que incluye diferencias de género, raza, cultura, orientación sexual, } \\
\text { discapacidad, edad, entre otras. } \\
\text { - En el nivel género, es igualdad entre hombres y mujeres; en el nivel } \\
\text { racial, se tiene todas las razas dentro de la empresa; en el ámbito } \\
\text { cultural y de nacionalidad, se cuenta con personal de Bolivia, Chile y } \\
\text { Colombia, además del peruano. }\end{array}$ \\
\hline Tecnología (1) & $\begin{array}{l}\text { Es respetar las diferencias que existen entre todos los empleados de } \\
\text { la empresa sin juzgarlas. Es otorgar las mismas oportunidades y con- } \\
\text { diciones sin importar de donde vengan o cómo son. }\end{array}$ \\
\hline Consumo masivo (1) & $\begin{array}{l}\text { Es una estrategia de negocio. Lo que se busca es lograr una ventaja } \\
\text { competitiva por medio del uso de la diversidad en la organización. }\end{array}$ \\
\hline
\end{tabular}

Fuente: Trabajos presentados por los alumnos María Elena Becerra, Marisel Coello, Gabriela Llerena, Jorge Moreno, Nikoska Nicolás, Paola Núñez, Katherine Rojas, Rosa María Rovegno y Alejandra Valdez para el Taller de Investigación «Diversidad como Estrategia de Gestión». Universidad del Pacífico, primer semestre de 2009.

Elaboración propia.

\section{Cuadro 2}

\section{Origen de la filosofía de diversidad}

\begin{tabular}{|l|l|l|}
\hline \multicolumn{1}{|c|}{ Empresas por sector } & \multicolumn{1}{c|}{ La filosofía de diversidad proviene de } \\
\hline Consultoras en auditoría (2) & • De la casa matriz (Reino Unido). Del Perú. \\
\hline Tecnología (1) & - De la casa matriz (Estados Unidos) \\
\hline Consumo masivo (1) & - De la casa matriz (Estados Unidos) \\
\hline
\end{tabular}

Fuente: Trabajos presentados por los alumnos María Elena Becerra, Marisel Coello, Gabriela Llerena, Jorge Moreno, Nikoska Nicolás, Paola Núñez, Katherine Rojas, Rosa María Rovegno y Alejandra Valdez para el Taller de Investigación «Diversidad como Estrategia de Gestión». Universidad del Pacífico, primer semestre de 2009.

Elaboración propia. 
Encargado de manejar temas de diversidad

No es una única área la que se encarga de gestionar la diversidad (véase el cuadro 3). En algunos casos, la responsabilidad recae en la gerencia de recursos humanos; en otros, en la propia gerencia general.

Ámbitos de la diversidad desarrollados en las empresas

El ámbito de la diversidad que se desarrolla principalmente en las empresas encuestadas es el de género (véase el cuadro 4). La razón, según la información de fuentes secundarias, es que la mujer en América Latina aún no tiene presencia representativa en los altos cargos directivos.

Principales ventajas de la diversidad para la empresa

Las empresas encuestadas consideran que los empleados y los clientes son los beneficiados, y que las prácticas de gestión de la diversidad contribuyen a retener el talento porque motivan al trabajador (véase el cuadro 5).

\section{Cuadro 3}

Responsable de la filosofía de diversidad

\begin{tabular}{|l|l|}
\hline \multicolumn{1}{|c|}{ Empresas por sector } & \multicolumn{1}{c|}{ La diversidad se gestiona desde } \\
\hline Consultoras en auditoría (2) & $\begin{array}{l}\text { - En el Perú, la Gerencia de Recursos Humanos. } \\
\text { - Cada uno de los miembros es responsable. }\end{array}$ \\
\hline Tecnología (1) & $\begin{array}{l}\text { En el ámbito global existe un área específica. En el Perú, la } \\
\text { Gerencia de Recursos Humanos. }\end{array}$ \\
\hline Consumo masivo (1) & - En el Perú, la Gerencia General. \\
\hline
\end{tabular}

Fuente: Trabajos presentados por los alumnos María Elena Becerra, Marisel Coello, Gabriela Llerena, Jorge Moreno, Nikoska Nicolás, Paola Núñez, Katherine Rojas, Rosa María Rovegno y Alejandra Valdez para el Taller de Investigación «Diversidad como Estrategia de Gestión». Universidad del Pacífico, primer semestre de 2009.

Elaboración propia.

\section{Cuadro 4}

\section{Ámbitos de la diversidad desarrollados en las empresas}

\begin{tabular}{|l|l|}
\hline \multicolumn{1}{|c|}{ Empresas por sector } & \multicolumn{1}{c|}{ Ámbitos de la diversidad } \\
\hline Consultoras en auditoría (2) & $\begin{array}{l}\text { - Diversidad de género. } \\
\text { - Ninguno en especial. }\end{array}$ \\
\hline Tecnología (1) & $\begin{array}{l}\text { - Diversidad de género, edad, orientación sexual, vida personal y } \\
\text { profesional y discapacidad. }\end{array}$ \\
\hline Consumo masivo (1) & $\begin{array}{l}\text { - Diversidad de género, estilos de pensamiento y origen del recluta- } \\
\text { miento. }\end{array}$ \\
\hline
\end{tabular}

Fuente: Trabajos presentados por los alumnos María Elena Becerra, Marisel Coello, Gabriela Llerena, Jorge Moreno, Nikoska Nicolás, Paola Núñez, Katherine Rojas, Rosa María Rovegno y Alejandra Valdez para el Taller de Investigación «Diversidad como Estrategia de Gestión». Universidad del Pacífico, primer semestre de 2009.

Elaboración propia. 


\section{Cuadro 5}

\section{Ventajas de la diversidad}

\begin{tabular}{|l|l|}
\hline \multicolumn{1}{|c|}{ Empresas por sector } & \multicolumn{1}{c|}{ Ventajas } \\
\hline Consultoras en auditoría (2) & $\begin{array}{l}\text { - Ofrece beneficios al trabajador porque desarrolla su potencial y } \\
\text { habilidades de interrelación; al cliente, porque cuenta con diver- } \\
\text { sidad de visiones y soluciones; y a los equipos de trabajo, porque } \\
\text { pueden generar mejores resultados. } \\
\text { - Permite balancear opiniones, considerar diversas perspectivas, } \\
\text { incluso de culturas distintas. }\end{array}$ \\
\hline Tecnología (1) & $\begin{array}{l}\text { - Permite retener al talento mediante la satisfacción de sus necesi- } \\
\text { dades. }\end{array}$ \\
\hline & - Motiva al personal, se siente más contento en la empresa. \\
\hline
\end{tabular}

Fuente: Trabajos presentados por los alumnos María Elena Becerra, Marisel Coello, Gabriela Llerena, Jorge Moreno, Nikoska Nicolás, Paola Núñez, Katherine Rojas, Rosa María Rovegno y Alejandra Valdez para el Taller de Investigación «Diversidad como Estrategia de Gestión». Universidad del Pacífico, primer semestre de 2009.

Elaboración propia.

\section{Principales desventajas de la diversidad para la empresa}

Dos de las cuatro empresas manifestaron que no existen desventajas (véase el cuadro 6). Sin embargo, las otras dos mencionaron como un problema la distorsión de la comunicación. Es relevante destacar que aun cuando se podrían identificar desventajas, las empresas son conscientes de la manera de superarlas.

\section{Políticas escritas sobre diversidad}

Dos de las empresas encuestadas poseen códigos escritos en los que se menciona el respeto a la diversidad y el derecho a la no discriminación (véase el cuadro 7). El hecho de tratar el tema de diversidad en el nivel de códigos de comportamiento de las empresas, es una muestra del grado de relevancia e interiorización del tema en la cultura organizacional.

\section{Cuadro 6}

\section{Desventajas de la diversidad}

\begin{tabular}{|l|l|}
\hline \multicolumn{1}{|c|}{ Empresas por sector } & \multicolumn{1}{c|}{ Desventajas } \\
\hline Consultoras en auditoría (2) & $\begin{array}{l}\text { - Ninguna. } \\
\text { - Ninguna. }\end{array}$ \\
\hline Tecnología (1) & \begin{tabular}{l} 
- La distorsión de la comunicación puede ser un problema. \\
\hline Consumo masivo (1)
\end{tabular} \\
& $\begin{array}{l}\text { L La comunicación. Talleres especializados contribuyen a } \\
\text { solucionar el problema. }\end{array}$ \\
\hline
\end{tabular}

Fuente: Trabajos presentados por los alumnos María Elena Becerra, Marisel Coello, Gabriela Llerena, Jorge Moreno, Nikoska Nicolás, Paola Núñez, Katherine Rojas, Rosa María Rovegno y Alejandra Valdez para el Taller de Investigación «Diversidad como Estrategia de Gestión». Universidad del Pacífico, primer semestre de 2009.

Elaboración propia. 


\section{Cuadro 7}

\section{Políticas escritas sobre diversidad}

\begin{tabular}{|l|l|}
\hline \multicolumn{1}{|c|}{ Empresas por sector } & \multicolumn{1}{c|}{ Desventajas } \\
\hline Consultoras en auditoría (2) & $\begin{array}{l}\text { - Código global de conducta reprueba toda práctica } \\
\text { discriminatoria. } \\
\text { - No. }\end{array}$ \\
\hline Tecnología (1) & - No. \\
\hline Consumo masivo (1) & - Código de ética. Políticas establecidas. \\
\hline
\end{tabular}

Fuente: Trabajos presentados por los alumnos María Elena Becerra, Marisel Coello, Gabriela Llerena, Jorge Moreno, Nikoska Nicolás, Paola Núñez, Katherine Rojas, Rosa María Rovegno y Alejandra Valdez para el Taller de Investigación «Diversidad como Estrategia de Gestión». Universidad del Pacífico, primer semestre de 2009.

Elaboración propia.

\section{Proyectos específicos sobre diversidad}

Las cuatro empresas poseen más de un mecanismo para fomentar la diversidad (véase el cuadro 8). El que destaca es el vinculado a brindar flexibilidad y balance de vida personal-profesional.

\section{Cuadro 8}

\section{Proyectos específicos sobre diversidad}

\begin{tabular}{|l|l|}
\hline \multicolumn{1}{|c|}{ Empresas por sector } & \multicolumn{1}{c|}{ Proyectos específicos } \\
\hline Consultoras en auditoría (2) & $\begin{array}{l}\text { - } \begin{array}{l}\text { Foro de mujeres, en el que las profesionales intercambian expe- } \\
\text { riencias y consejos. }\end{array} \\
\text { - Se trabajan temas relativos a la flexibilidad y el balance personal- } \\
\text { profesional. Por ejemplo, los padres tienen tres días libres ante el } \\
\text { nacimiento de un hijo; una madre puede tomar una hora de descan- } \\
\text { so durante un año; se entregan computadoras portátiles para que } \\
\text { se trabaje desde la casa. }\end{array}$ \\
\hline Tecnología (1) & $\begin{array}{l}\text { Existen los Diversity Net Groups, grupos de personas que se aso- } \\
\text { cian por tener algo en común. Se trabaja el tema de flexibilidad, } \\
\text { por ejemplo, con teletrabajo y talleres para los hijos, los cuales se } \\
\text { ofrecen en la empresa. }\end{array}$ \\
\hline Consumo masivo (1) & $\begin{array}{l}\text { Se está trabajando en el origen del reclutamiento. Se recluta de } \\
\text { distintas ciudades y no solo de Lima. }\end{array}$ \\
\hline
\end{tabular}

Fuente: Trabajos presentados por los alumnos María Elena Becerra, Marisel Coello, Gabriela Llerena, Jorge Moreno, Nikoska Nicolás, Paola Núñez, Katherine Rojas, Rosa María Rovegno y Alejandra Valdez para el Taller de Investigación «Diversidad como Estrategia de Gestión». Universidad del Pacífico, primer semestre de 2009.

Elaboración propia. 
Cabe mencionar que en el Perú se ha aprobado otorgar una licencia por paternidad. El 10 de setiembre de 2009, el Congreso aprobó que los trabajadores de la actividad pública y privada y los miembros de las Fuerzas Armadas y de la Policía Nacional gozarán de cuatro días hábiles consecutivos bajo la modalidad de licencia remunerada. El inicio de la licencia lo determinará el trabajador y deberá estar comprendida entre el día del alumbramiento y el día que le den de alta a la madre $o$ al hijo. La finalidad de la medida es promover y fortalecer el desarrollo de la familia.

\section{Diversidad de género}

En todas las empresas encuestadas es evidente que las posiciones las ocupan quienes lo ameriten, en función de sus cualidades y experiencia y no de su género (véase el cuadro 9). Es importante mencionar que los niveles salariales para los hombres y las mujeres son los mismos en estas empresas.

\section{Cuadro 9}

Diversidad de género

\begin{tabular}{|c|c|}
\hline Empresas por sector & Diversidad de género \\
\hline Consultoras en auditoría ( 1 ) & $\begin{array}{l}\text { - La brecha de género identificada no obedece a falta de oportunida- } \\
\text { des de desarrollo, sino a la alta carga de trabajo frente a las obliga- } \\
\text { ciones personales y familiares. } \\
\text { - En todos los puestos, ambos géneros tienen la misma posibilidad de } \\
\text { reclutamiento y desarrollo de línea de carrera. En los últimos años, } \\
\text { el número de socios y gerentes mujeres ha aumentado. Las políti- } \\
\text { cas salariales carecen de rasgos de disparidad. } \\
\text { - Se busca mantener la equidad en la contratación del número de } \\
\text { hombres y mujeres. }\end{array}$ \\
\hline Tecnología (1) & $\begin{array}{l}\text { - La contratación se realiza considerando el perfil de la persona que } \\
\text { cubrirá el puesto, no el género. } \\
\text { - Es un tema de respeto que ambos géneros tengan las mismas condi- } \\
\text { ciones salariales. }\end{array}$ \\
\hline Consumo masivo (1) & $\begin{array}{l}\text { - Se busca mantener el equilibrio en el número de hombres y mujeres } \\
\text { contratados. } \\
\text { - La contratación se realiza considerando el perfil de la persona que } \\
\text { cubrirá el puesto, no el género. } \\
\text { - No existe diferencia en los sueldos y beneficios por género. }\end{array}$ \\
\hline
\end{tabular}

Fuente: Trabajos presentados por los alumnos María Elena Becerra, Marisel Coello, Gabriela Llerena, Jorge Moreno, Nikoska Nicolás, Paola Núñez, Katherine Rojas, Rosa María Rovegno y Alejandra Valdez para el Taller de Investigación «Diversidad como Estrategia de Gestión». Universidad del Pacífico, primer semestre de 2009.

Elaboración propia. 
Diversidad de edad

Las empresas encuestadas no discriminan por el factor edad (véase el cuadro 10). Tanto los jóvenes como los mayores pueden ocupar las diversas posiciones, pues lo que se valora es el perfil adecuado para el puesto.

\section{Diversidad en el Perú}

Las empresas encuestadas coinciden en señalar que en el Perú el tema de la diversidad no está muy difundido y que la diversidad de género debería trabajarse con prioridad (véase el cuadro 11). Asimismo, indican que el origen en la mayoría de empresas involucradas en el tema es extranjero.

\section{Cuadro 10}

Diversidad de edad

\begin{tabular}{|c|c|}
\hline Empresas por sector & Diversidad de edad \\
\hline Consultoras en auditoría ( 1 ) & $\begin{array}{l}\text { - En la contratación se valora el potencial y la experiencia de los } \\
\text { postulantes. } \\
\text { - El desarrollo de carrera se centra en la meritocracia. Se puede } \\
\text { ascender a gerente, aproximadamente, a los } 30 \text { años. } \\
\text { - Se puede contratar gente mayor, pues se valora su experiencia. }\end{array}$ \\
\hline Tecnología (1) & $\begin{array}{l}\text { - Se valora la experiencia. El personal joven con las cualidades ne- } \\
\text { cesarias puede ocupar cargos gerenciales. }\end{array}$ \\
\hline Consumo masivo (1) & $\begin{array}{l}\text { - La empresa recluta a jóvenes terminando su carrera y promueve la } \\
\text { línea de carrera interna. } \\
\text { - Los jóvenes con el perfil requerido pueden ocupar cargos } \\
\text { gerenciales. }\end{array}$ \\
\hline
\end{tabular}

Fuente: Trabajos presentados por los alumnos María Elena Becerra, Marisel Coello, Gabriela Llerena, Jorge Moreno, Nikoska Nicolás, Paola Núñez, Katherine Rojas, Rosa María Rovegno y Alejandra Valdez para el Taller de Investigación «Diversidad como Estrategia de Gestión». Universidad del Pacífico, primer semestre de 2009.

Elaboración propia.

\section{Cuadro 11}

\section{Diversidad en el Perú}

\begin{tabular}{|l|l|}
\hline \multicolumn{1}{|c|}{ Empresas por sector } & \multicolumn{1}{c|}{ Diversidad en el Perú } \\
\hline Consultoras en auditoría (2) & $\begin{array}{r}\text { - La diversidad de género necesita trabajarse. Las mujeres aún no } \\
\text { tienen representatividad en los niveles gerenciales y de liderazgo en } \\
\text { la economía nacional. La probabilidad de que el salario de una mu- } \\
\text { jer sea menor que el de un hombre es muy alta. }\end{array}$ \\
\hline Tecnología (1) & $\begin{array}{l}\text { En el Perú aún no se ha desarrollado este tema. Es un tema que no } \\
\text { se ha internalizado como una fuente de ventaja competitiva. }\end{array}$ \\
\hline Consumo masivo (1) & $\begin{array}{l}\text { Es un tema poco manejado en el Perú, excepto por las que también } \\
\text { tienen actividades en el extranjero. La tendencia a entender real- } \\
\text { mente la importancia de la diversidad es creciente. }\end{array}$ \\
\hline
\end{tabular}

Fuente: Trabajos presentados por los alumnos María Elena Becerra, Marisel Coello, Gabriela Llerena, Jorge Moreno, Nikoska Nicolás, Paola Núñez, Katherine Rojas, Rosa María Rovegno y Alejandra Valdez para el Taller de Investigación «Diversidad como Estrategia de Gestión». Universidad del Pacífico, primer semestre de 2009.

Elaboración propia. 


\section{Conclusiones}

- En el Perú, la gestión de la diversidad aún se encuentra en una etapa inicial. Las empresas que realmente ven a la diversidad como una ventaja competitiva forman parte, en su mayoría, de empresas cuya filosofía de la diversidad proviene de la casa matriz. Es interesante mencionar que adicionalmente al estudio realizado, el 13 de octubre de 2009 se hizo público en el diario El Comercio un informe del BID para América Latina, en el que se señala que el mercado laboral peruano está entre los más discriminatorios. La brecha salarial por sexo llega a $19,3 \%$ y a $21 \%$ por el grupo étnico.

- La principal preocupación de las empresas latinoamericanas, en cuanto a diversidad, es la inclusión de la mujer en el ámbito laboral en iguales condiciones que los varones.

- La búsqueda de personas para ocupar las diferentes posiciones en las empresas encuestadas se realiza en función del cumplimiento del perfil requerido, sin importar su género o edad.

- Las empresas peruanas encuestadas identifican ventajas en gestionar la diversidad. Estas ventajas no son solo internas sino externas, hacia el cliente.

- La gestión de la diversidad en las empresas encuestadas recae, principalmente, en las áreas de Recursos Humanos. Son estas áreas las que desarrollan diversos proyectos para promover la diversidad, entre los que destacan los relacionados con brindar flexibilidad laboral. 


\section{Referencias}

ABURDENE, P. y J. NAISBITT

1993 Megatendencias de la mujer. Colombia: Grupo editorial Norma.

ALLES, M.

2000 Mujeres, trabajo y autoempleo. Otro camino frente al desempleo y desarrollo de carrera. Editorial Granica.

BARBERA, E.; A. RAMOS, M. SARRIÓ y C. CANDELA

2002 «Más allá del «techo de cristal». Diversidad de género». En: Revista del Ministerio de Trabajo e Inmigración, № 40, pp. 55-68.

BARBERA, E.; C. CANDELA, J. CHORRO, A. RAMOS, M. SARRIÓ, J. SEVILLA y T. YEVES

s.f. «Rompiendo el Techo de Cristal: Ios beneficios de la diversidad de género en los equipos de dirección». Proyecto de Investigación. Valencia: Universitat de València.

BURMAN, E.

2008 «Managing Cultural Diversity in a Global World». En: Human Resources for Today"s Workplace. Fecha de consulta: 30/09/2009. <http://www.workinfo.com/free/downloads/ 299.htm>.

CHEMERS, M.; S. OSKAMP y M. CONSTANZO

1995 Diversity in Organizations: New Perspectives for a Changing Workplace. California: SAGE Publications Inc.

COX, T. y S. BLAKE

1991 «Managing cultural diversity: Implications for organization». En: Academy of Management Executive, vol. 5. № 3, pp. 45-56.

DE ANCA, C.

2008 «La gestión de la diversidad en la organización global». En: Conocimiento y Dirección, $\mathrm{N}^{\circ}$ 77, pp. 88-91.

2004 Diversidad de género y cultura en la gestión global. Madrid: Departamento de Publicaciones del Instituto de Empresa.
GARCÍA, M. L.

2008 «Equilibrio de género. Auditoría de igualdad en las empresas». En: Mujeres en Red, periódico virtual. Fecha de consulta: 02/10/ 2009. <http://www.mujeresenred.net/ spip.php?article1374>.

\section{HALLADAY, J. y A. THOMAS}

2002 The Rise of Women Entrepreneurs: People, Processes and Global Trends. Westport, CT: Quorum Books.

\section{MANAUT, S.}

2006 «La diversidad generacional demanda a las empresas una respuesta variada». En: Instituto Europeo para la Gestión de la Diversidad (IEGD). Fecha de consulta: 28/09/2009. <http://www.tendencias21.net/La-diversidadgeneracional-demanda-a-las-empresas-unarespuesta-variada_a975.html>.

MAXFIELD, S.

2007 "Linking business's gender and diversity practices with corporate citizenship: Implications for Latin America». En: Academia, Revista Latinoamericana de Administración, $N^{\circ} 38$, pp. 65-80.

MAYO, M.

2002 «La gestión de la diversidad». En: BONACHE, J. y P. IBARRA (eds.). Dirección estratégica de personas. España: Editorial Prentice Hall.

MONDY, W. y R. NOE

2005 Administración de recursos humanos. Novena edición. México: Editorial Pearson Prentice Hall.

MORALES, C.

2006 «Las prácticas eficaces en la gestión de la diversidad». En: Harvard Deusto Business Review, No 142, pp. 64-69.

\section{OVALLE, I. y L. MARCHANT}

2005 «Diversidad cultural y desarrollo organizacional». En: Biblioteca Virtual de Economía. Fecha de consulta: 30/09/2009. <http://www.eumed.net/libros/2005/Imr/ 10.htm>. 
PIN, J.; P. GARCÍA y A. GALIFFA

2007 Libro blanco sobre la gestión de la diversidad en las empresas españolas: retos, oportunidades y buenas prácticas. España: IESE.

PIRAS, C.

2006 Mujeres y trabajo en América Latina. Desafíos para las políticas públicas. Washington, D. C.: Banco Interamericano de Desarrollo.

RAMOS, María Amparo

2005 «Liderazgo femenino: diversidad de género como estrategia de cambio». Jornadas de Difusión y Estudios de Género en Palencia. España, octubre.

ROBBINS, S. y M. COULTER

2005 Administración. Octava edición. México: Pearson Prentice Hall.

ROSCIGNO, V.; S. MONG, R. BYRON y G. TESTER 2007 «Age Discrimination, Social Closure and Employment». En: Social Forces, vol. 86, N 1, pp. 313-334.
SIN AUTOR

2009 «Mercado laboral peruano está entre los más discriminatorios». En: El Comercio. 13 de octubre. B1.

SUSAETA, L. y J. NAVA

2005 «La diversidad cultural como fuente de ventaja competitiva. Una aplicación a la empresa multinacional». En: Cuadernos de Estudios Empresariales, № 15, pp. 153-177.

THOMAS, D. y E. Robin

1999 «Cómo hacer que las diferencias importen. Un Nuevo modelo para gestionar la diversidad». En: Harvard Business Review, Dirigir personas en la empresa. España, Barcelona: Ediciones Deusto, pp. 137-173.

WALKER, A.

1999 «Combating Age Discrimination at the Workplace». En: Experimental Aging Research, $N^{\circ} 25$, pp. 367-377. 